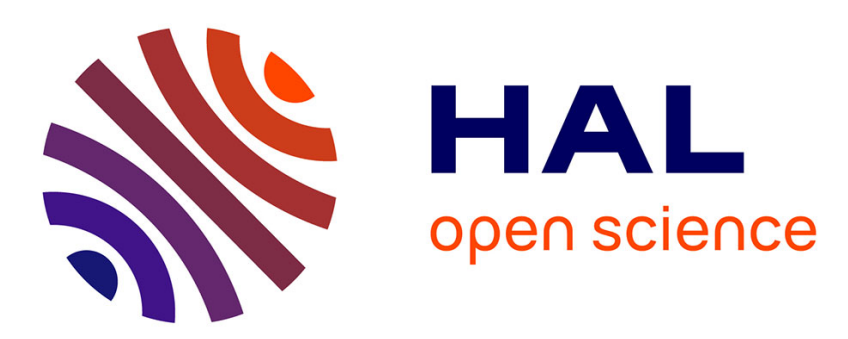

\title{
Existence of solutions of a dynamic Signorini's problem with nonlocal friction in viscoelasticity \\ Marius Cocou
}

\section{To cite this version:}

Marius Cocou. Existence of solutions of a dynamic Signorini's problem with nonlocal friction in viscoelasticity. Zeitschrift für Angewandte Mathematik und Physik, 2002, 53 (6), pp.1099-1109. 10.1007/PL00012615 . hal-00166689

\section{HAL Id: hal-00166689 \\ https://hal.science/hal-00166689}

Submitted on 8 Aug 2007

HAL is a multi-disciplinary open access archive for the deposit and dissemination of scientific research documents, whether they are published or not. The documents may come from teaching and research institutions in France or abroad, or from public or private research centers.
L'archive ouverte pluridisciplinaire HAL, est destinée au dépôt et à la diffusion de documents scientifiques de niveau recherche, publiés ou non, émanant des établissements d'enseignement et de recherche français ou étrangers, des laboratoires publics ou privés. 


\title{
Existence of solutions of a dynamic Signorini's problem with nonlocal friction in viscoelasticity
}

\author{
Marius Cocou
}

Laboratoire de Mécanique et d'Acoustique - C.N.R.S. 31, chemin Joseph Aiguier

13402 Marseille Cedex 20 and Université de Provence U.F.R. - M.I.M., Marseille, France

e-mail: cocou@lma.cnrs-mrs.fr

Dedicated to the memory of Professor Eugen Soós, my Teacher

\begin{abstract}
A dynamic unilateral contact problem with nonlocal friction for a viscoelastic body satisfying a Kelvin-Voigt law is studied. Using a penalty method and compactness results, the existence of a weak solution of this problem is proved.
\end{abstract}

Mathematics Subject Classification (2000). 35Q99, 35R35, 49J40, 74A55, 74D05, 74H20.

Keywords. Dynamic problems, unilateral contact, nonlocal friction, viscoelastic body, existence of solutions.

\section{Introduction}

In this paper, we consider a dynamic Signorini's problem with a nonlocal friction law for a viscoelastic body of Kelvin-Voigt type.

G. Duvaut and J. L. Lions [2] studied dynamic and quasistatic contact problems with Tresca's (or given) friction law involving linearly elastic and viscoelastic bodies. Dynamic bilateral contact with nonlocal friction has been analyzed very recently by K. L. Kuttler and M. Shillor [7].

J. A. C. Martins and J. T. Oden [10] proved the existence and uniqueness, for a viscoelastic material, of a solution to dynamic contact problems with normal compliance. Similarly, I. Figueiredo and L. Trabucho [3] extended these results to a class of (thermovisco)elastic problems. K. L. Kuttler [6] has weakened the restrictions on the coefficients of the normal compliance law.

More recently, M. Cocou and J. M. Ricaud [1], E. Pratt and J. M. Ricaud [12] have studied abstract implicit evolution inequalities with applications to dynamic contact problems for viscoelastic bodies.

An existence and uniqueness result for the wave equation with unilateral boundary conditions for a half-space was proved by G. Lebeau and M. Schatzman [8]. Existence of solutions in the case of a smooth bounded domain subjected to uni- 
lateral conditions on its boundary has been proved by J. U. Kim [5] for the wave equation and by J. Muñoz-Rivera and R. Racke [11] for a thermoelastic radially symmetric body.

The dynamic Signorini's frictionless contact problem for viscoelastic materials with singular memory has been considered by J. Jarušek [4] who studied also the case of given friction.

The paper is organized as follows. Section 2 is devoted to strong and weak formulations of the problem. In Section 3 a penalized problem is investigated by using an abstract existence and uniqueness result for the solution of some implicit evolution inequalities. In Section 4 several estimates on the penalized solutions are established which allow to pass to the limit and to obtain a solution of the dynamic Signorini's problem with nonlocal friction.

\section{Classical and variational formulations}

Let us consider a viscoelastic body that initially occupies a bounded domain $\Omega$ in $\mathbb{R}^{d}, d=2,3$, with a sufficiently smooth boundary $\Gamma=\partial \Omega$. This boundary is divided into three disjoint and open parts $\Gamma=\bar{\Gamma}_{u} \cup \bar{\Gamma}_{f} \cup \bar{\Gamma}_{c}$. The body is subjected to volume forces of density $\mathbf{F}_{\mathbf{1}}$, prescribed zero displacements and tractions $\mathbf{F}_{\mathbf{2}}$ on the parts $\Gamma_{u}$ and $\Gamma_{f}$ respectively. To simplify the arguments we assume that $\operatorname{mes}\left(\Gamma_{u}\right)>0$ and that the part $\Gamma_{c}$, where the body is in unilateral contact with a fixed foundation, is a bounded contact zone.

We shall denote by $\boldsymbol{u}=\left(u_{i}\right)$ the displacement field, by $\boldsymbol{\sigma}=\left(\sigma_{i j}\right)$ the stress tensor and by $\boldsymbol{\epsilon}=\left(\epsilon_{i j}\right)=\frac{1}{2}\left(u_{i, j}+u_{j, i}\right)$ the strain tensor, for $i, j \in\{1, . ., d\}$. We denote respectively by $\mathbf{A}=\left(a_{i j k l}\right)$ and $\mathbf{B}=\left(b_{i j k l}\right)$ the elasticity and viscosity tensors. Suppose that $\mathbf{A}$ and $\mathbf{B}$ satisfy the usual properties of ellipticity and symmetry and that $a_{i j k l}$ and $b_{i j k l}$ are in $\mathrm{W}^{1, \infty}\left(\mathbb{R}^{d}\right), \forall i, j, k, l=1, \ldots, d$. We shall adopt the usual notations for the normal and tangential components of displacement vector and stress vector:

$$
\boldsymbol{u}=u_{N} \boldsymbol{n}+\boldsymbol{u}_{\mathrm{T}}, \quad u_{N}=u_{i} n_{i}, \quad \boldsymbol{\sigma} \boldsymbol{n}=\sigma_{N} \boldsymbol{n}+\boldsymbol{\sigma}_{\mathrm{T}}, \quad \sigma_{N}=\sigma_{i j} n_{i} n_{j},
$$

where $\boldsymbol{n}=\left(n_{i}\right)$ is the outward normal unit vector to the boundary. The notation |.| denotes the absolute value when applied to a scalar and the euclidian norm, corresponding to the scalar product ".", when applied to an element of $\mathbb{R}^{d}$.

Under the hypothesis of small displacements, the classical formulation of the dynamic Signorini's problem with nonlocal friction in viscoelasticity is as follows.

Problem $\mathrm{P}_{0}$ : Find $\boldsymbol{u}=\boldsymbol{u}(t, \mathbf{x})$ such that $\boldsymbol{u}(0)=\boldsymbol{u}_{\mathbf{0}}, \dot{\boldsymbol{u}}(0)=\boldsymbol{u}_{\mathbf{1}}$ and for all $\mathrm{t}$ in $] 0, \mathrm{~T}[$

$$
\begin{aligned}
& \operatorname{div} \boldsymbol{\sigma}+\mathbf{F}_{\mathbf{1}}=\ddot{\boldsymbol{u}} \quad \text { in } \Omega, \\
& \boldsymbol{\sigma}=\mathbf{A} \boldsymbol{\epsilon}(\boldsymbol{u})+\mathbf{B} \boldsymbol{\epsilon}(\dot{\boldsymbol{u}}), \\
& \boldsymbol{u}=\mathbf{0} \quad \text { on } \Gamma_{u}, \quad \boldsymbol{\sigma} \boldsymbol{n}=\mathbf{F}_{\mathbf{2}} \quad \text { on } \Gamma_{f},
\end{aligned}
$$




$$
\begin{aligned}
& u_{N} \leq 0, \quad \sigma_{N} \leq 0, \quad u_{N} \sigma_{N}=0 \quad \text { on } \Gamma_{c}, \\
& \left|\boldsymbol{\sigma}_{\mathrm{T}}\right| \leq \mu\left|(\mathcal{R} \boldsymbol{\sigma})_{N}\right| \text { and }\left\{\begin{array}{l}
\left|\boldsymbol{\sigma}_{\mathrm{T}}\right|<\mu\left|(\mathcal{R} \boldsymbol{\sigma})_{N}\right| \Rightarrow \dot{\boldsymbol{u}}_{\mathrm{T}}=\mathbf{0} \\
\left|\boldsymbol{\sigma}_{\mathrm{T}}\right|=\mu\left|(\mathcal{R} \boldsymbol{\sigma})_{N}\right| \Rightarrow \exists \lambda \geq 0, \dot{\boldsymbol{u}}_{\mathrm{T}}=-\lambda \boldsymbol{\sigma}_{\mathrm{T}},
\end{array} \quad \text { on } \Gamma_{c},\right.
\end{aligned}
$$

where $\mu \in \mathrm{L}^{\infty}\left(\Gamma_{c}\right), \mu \geq 0$ a.e. on $\Gamma_{c}$, is the friction coefficient and $(\mathcal{R} \boldsymbol{\sigma})_{N}$ is a regularization of the normal contact force which will be described below. Note that we can also consider non zero displacements on $\Gamma_{u}$ or prescribed tangential velocities for the foundation.

The functional framework for a weak formulation of this problem is as follows:

$$
\begin{aligned}
& \boldsymbol{H}^{s}(\Omega)=\left[H^{s}(\Omega)\right]^{d}, s \in \mathbb{R}, \mathrm{V}=\left\{\boldsymbol{v} \in \boldsymbol{H}^{1}(\Omega) ; \gamma \boldsymbol{v}=\mathbf{0} \text { a.e. on } \Gamma_{u}\right\}, \\
& \mathrm{K}=\left\{\boldsymbol{v} \in \mathrm{V} ;(\gamma v)_{N} \leq 0 \text { a.e. on } \Gamma_{c}\right\}, \boldsymbol{H}_{0}^{1}(\Omega)=\left[H_{0}^{1}(\Omega)\right]^{d}, H=\left[\mathrm{L}^{2}(\Omega)\right]^{d}, \\
& \mathbf{L}^{2}\left(\Gamma_{c}\right)=\left[\mathrm{L}^{2}\left(\Gamma_{c}\right)\right]^{d}, \mathbf{L}^{2}\left(\Gamma_{f}\right)=\left[\mathrm{L}^{2}\left(\Gamma_{f}\right)\right]^{d} .
\end{aligned}
$$

We shall use the notations $||=.|\cdot|_{H},\|\|=.\|\cdot\|_{\mathrm{V}}$ and we shall omit $\gamma$ in order to simplify the notation for the trace mapping.

Assume that

$$
\boldsymbol{u}_{\mathbf{0}} \in \mathrm{K}, \boldsymbol{u}_{\mathbf{1}} \in \mathrm{V}, \mathbf{F}_{\mathbf{1}} \in \mathrm{W}^{1, \infty}(0, \mathrm{~T} ; H) \text { and } \mathbf{F}_{\mathbf{2}} \in \mathrm{W}^{1, \infty}\left(0, \mathrm{~T} ; \mathbf{L}^{2}\left(\Gamma_{f}\right)\right) .
$$

Using the previous hypotheses, let us denote by $\mathbf{L}$ the element of $\mathrm{V}$ defined by

$$
\langle\mathbf{L}, \boldsymbol{v}\rangle=\int_{\Omega} \mathbf{F}_{\mathbf{1}} \cdot \boldsymbol{v} d x+\int_{\Gamma_{f}} \mathbf{F}_{\mathbf{2}} \cdot \boldsymbol{v} d s, \forall \boldsymbol{v} \in \mathrm{V},
$$

where $\langle.,$.$\rangle denotes the scalar product on \mathrm{V}$.

Suppose that $\mathcal{R}:\left[\mathrm{L}_{s}^{2}(\Omega)\right]^{d^{2}} \rightarrow\left[H^{1}(\Omega)\right]^{d^{2}}$, where $\left[\mathrm{L}_{s}^{2}(\Omega)\right]^{d^{2}}=\left\{\boldsymbol{\tau} \in\left[\mathrm{L}^{2}(\Omega)\right]^{d^{2}}\right.$; $\left.\boldsymbol{\tau}=\left(\tau_{i j}\right), \tau_{i j}=\tau_{j i}, i, j=1, \ldots, d\right\}$, is a linear and continuous operator which has the following properties: $\left(\mathcal{R} \boldsymbol{\sigma}\left(\boldsymbol{u}_{\mathbf{0}}, \boldsymbol{u}_{\mathbf{1}}\right)\right)_{N}=0$ and

$$
\text { if } \dot{\boldsymbol{u}}^{k} \rightarrow \dot{\boldsymbol{u}} \text { in } \mathrm{L}^{2}(0, \mathrm{~T} ; H) \text {, then }\left(\mathcal{R} \boldsymbol{\sigma}^{k}\right)_{N} \rightarrow(\mathcal{R} \boldsymbol{\sigma})_{N} \text { in } \mathrm{L}^{2}\left(0, \mathrm{~T} ; \mathrm{L}^{2}\left(\Gamma_{c}\right)\right) \text {. }
$$

An operator satisfying these conditions can be obtained by extending $\boldsymbol{u}$ and $\dot{\boldsymbol{u}}$ to all of $\mathbb{R}^{n}$ and by using the convolution with a smooth function, which gives an averaged normal stress, see for example [7].

We consider the following weak formulation of problem $\mathrm{P}_{0}$.

Problem P : Find $\boldsymbol{u} \in \mathrm{W}^{1,2}(0, \mathrm{~T} ; \mathrm{V}) \cap \mathrm{C}^{1}\left([0, \mathrm{~T}] ; \boldsymbol{H}^{-1 / 2}(\Omega)\right)$, such that $\boldsymbol{u}(0)=$ $\boldsymbol{u}_{\mathbf{0}}, \dot{\boldsymbol{u}}(0)=\boldsymbol{u}_{\mathbf{1}}, \boldsymbol{u}(t) \in \mathrm{K}$ for all $\mathrm{t}$ in $] 0, \mathrm{~T}[$ and

$$
\begin{aligned}
& \langle\dot{\boldsymbol{u}}(\mathrm{T}), \boldsymbol{v}(\mathrm{T})-\boldsymbol{u}(\mathrm{T})\rangle_{H^{-1 / 2}, \boldsymbol{H}^{1 / 2}}-\left(\boldsymbol{u}_{\mathbf{1}}, \boldsymbol{v}(0)-\boldsymbol{u}_{\mathbf{0}}\right) \\
& -\int_{0}^{\mathrm{T}}(\dot{\boldsymbol{u}}, \dot{\boldsymbol{v}}-\dot{\boldsymbol{u}}) d t+\int_{0}^{\mathrm{T}} a(\boldsymbol{u}, \boldsymbol{v}-\boldsymbol{u}) d t+\int_{0}^{\mathrm{T}} b(\dot{\boldsymbol{u}}, \boldsymbol{v}-\boldsymbol{u}) d t \\
& -\int_{0}^{\mathrm{T}} \int_{\Gamma_{c}} \mu\left|(\mathcal{R} \boldsymbol{\sigma})_{N}\right|\left(\left|\boldsymbol{v}_{\mathrm{T}}-\boldsymbol{u}_{\mathrm{T}}+\dot{\boldsymbol{u}}_{\mathrm{T}}\right|-\left|\dot{\boldsymbol{u}}_{\mathrm{T}}\right|\right) d s d t \geq \int_{0}^{\mathrm{T}}\langle\mathbf{L}, \boldsymbol{v}-\boldsymbol{u}\rangle d t \\
& \forall \boldsymbol{v} \in \mathrm{L}^{\infty}(0, \mathrm{~T} ; \mathrm{V}) \cap \mathrm{W}^{1,2}(0, \mathrm{~T} ; H) \text { with } \boldsymbol{v}(t) \in \mathrm{K} \text { for all } t \in[0, \mathrm{~T}],
\end{aligned}
$$


where $\quad a(\boldsymbol{v}, \boldsymbol{w})=\int_{\Omega} a_{i j k l} v_{i, j} w_{k, l} d x, \quad b(\boldsymbol{v}, \boldsymbol{w})=\int_{\Omega} b_{i j k l} v_{i, j} w_{k, l} d x$, $\forall \boldsymbol{v}, \boldsymbol{w} \in \mathrm{V},\langle., .\rangle_{\boldsymbol{H}^{-1 / 2}, \boldsymbol{H}^{1 / 2}}$ denotes the duality pairing between $\boldsymbol{H}^{-1 / 2}(\Omega)$ and $\boldsymbol{H}^{1 / 2}(\Omega)$ and $(.,$.$) is the usual scalar product on H$.

The equivalence between $\mathrm{P}_{0}$ and $\mathrm{P}$ can be easily proved by using Green's formula and an integration by parts.

We assume that the initial conditions $\boldsymbol{u}_{\mathbf{0}}, \boldsymbol{u}_{\mathbf{1}}$ satisfy the following compatibility condition : there exists $\boldsymbol{l} \in H$ such that

$$
a\left(\boldsymbol{u}_{\mathbf{0}}, \boldsymbol{v}\right)+b\left(\boldsymbol{u}_{\mathbf{1}}, \boldsymbol{v}\right)=\langle\mathbf{L}(0), \boldsymbol{v}\rangle+(\boldsymbol{l}, \boldsymbol{v}) \quad \forall \boldsymbol{v} \in \mathrm{V} .
$$

The existence of a solution of Problem $\mathrm{P}$ will be proven by using a penalty method.

\section{The penalized problem}

Let us consider the following strong formulation of the penalized problem, for $\varepsilon>0$, which can be seen as a dynamic contact problem with a normal compliance law.

Problem $\mathrm{P}_{0 \varepsilon}$ : Find $\boldsymbol{u}_{\varepsilon}=\boldsymbol{u}_{\varepsilon}(t, \mathbf{x})$ such that $\boldsymbol{u}_{\varepsilon}(0)=\boldsymbol{u}_{\mathbf{0}}, \dot{\boldsymbol{u}}_{\varepsilon}(0)=\boldsymbol{u}_{\mathbf{1}}$, and for all $\mathrm{t}$ in $] 0, \mathrm{~T}[$

$$
\begin{aligned}
& \text { div } \boldsymbol{\sigma}+\mathbf{F}_{\mathbf{1}}=\ddot{\boldsymbol{u}}_{\varepsilon} \quad \text { in } \Omega, \\
& \boldsymbol{\sigma}=\mathbf{A} \boldsymbol{\epsilon}\left(\boldsymbol{u}_{\varepsilon}\right)+\mathbf{B} \boldsymbol{\epsilon}\left(\dot{\boldsymbol{u}}_{\varepsilon}\right), \\
& \boldsymbol{u}_{\varepsilon}=\mathbf{0} \quad \text { on } \Gamma_{u}, \quad \boldsymbol{\sigma} \boldsymbol{n}=\mathbf{F}_{\mathbf{2}} \quad \text { on } \Gamma_{f}, \\
& \sigma_{N}=-\frac{1}{\varepsilon}\left(u_{\varepsilon N}\right)_{+} \quad \text { on } \Gamma_{c}, \\
& \left|\boldsymbol{\sigma}_{\mathrm{T}}\right| \leq \mu\left|(\mathcal{R} \boldsymbol{\sigma})_{N}\right| \text { and }\left\{\begin{array}{l}
\left|\boldsymbol{\sigma}_{\mathrm{T}}\right|<\mu\left|(\mathcal{R} \boldsymbol{\sigma})_{N}\right| \Rightarrow \dot{\boldsymbol{u}}_{\varepsilon \mathrm{T}}=\mathbf{0} \\
\left|\boldsymbol{\sigma}_{\mathrm{T}}\right|=\mu\left|(\mathcal{R} \boldsymbol{\sigma})_{N}\right| \Rightarrow \exists \lambda \geq 0, \dot{\boldsymbol{u}}_{\varepsilon \mathrm{T}}=-\lambda \boldsymbol{\sigma}_{\mathrm{T}} .
\end{array}\right.
\end{aligned}
$$

We shall analyze the following variational formulation of penalized problem.

Problem $\mathrm{P}_{\varepsilon}:$ Find $\boldsymbol{u}_{\varepsilon} \in \mathrm{W}^{1,2}(0, \mathrm{~T} ; \mathrm{V}) \cap \mathrm{W}^{2,2}(0, \mathrm{~T} ; H)$, such that $\boldsymbol{u}_{\varepsilon}(0)=$ $\boldsymbol{u}_{\mathbf{0}}, \dot{\boldsymbol{u}}_{\varepsilon}(0)=\boldsymbol{u}_{\mathbf{1}}$, and for almost all $\left.t \in\right] 0, \mathrm{~T}[$,

$$
\begin{gathered}
\left(\ddot{\boldsymbol{u}}_{\varepsilon}, \boldsymbol{v}-\dot{\boldsymbol{u}}_{\varepsilon}\right)+a\left(\boldsymbol{u}_{\varepsilon}, \boldsymbol{v}-\dot{\boldsymbol{u}}_{\varepsilon}\right)+b\left(\dot{\boldsymbol{u}}_{\varepsilon}, \boldsymbol{v}-\dot{\boldsymbol{u}}_{\varepsilon}\right)+\int_{\Gamma_{c}} \frac{1}{\varepsilon}\left(u_{\varepsilon N}\right)_{+}\left(v_{N}-\dot{u}_{\varepsilon N}\right) d s \\
+\int_{\Gamma_{c}} \mu\left|(\mathcal{R} \boldsymbol{\sigma})_{N}\right|\left(\left|\boldsymbol{v}_{\mathrm{T}}\right|-\left|\dot{\boldsymbol{u}}_{\varepsilon \mathrm{T}}\right|\right) d s \geq\left\langle\mathbf{L}, \boldsymbol{v}-\dot{\boldsymbol{u}}_{\varepsilon}\right\rangle \quad \forall \boldsymbol{v} \in \mathrm{V} .
\end{gathered}
$$

The existence of a solution of this problem can be obtained, for example, as application of existence results for set-valued pseudomonotone evolution inclusions, as developed recently by K.L. Kuttler and M. Shillor [7]. This method enables 
us, under less regular conditions on $\boldsymbol{u}_{\mathbf{1}}$ and $\mathbf{L}$, to prove the existence of a solution having the following regularity : $\boldsymbol{u}_{\varepsilon} \in \mathrm{W}^{1,2}(0, \mathrm{~T} ; \mathrm{V})$ and $\ddot{\boldsymbol{u}}_{\varepsilon} \in \mathrm{L}^{2}\left(0, \mathrm{~T} ; \mathrm{V}^{\prime}\right)$. Suitable estimates, which can be calculated if one assumes additional regularity properties of $\boldsymbol{u}_{\mathbf{1}}, \mathbf{L}$ and the compatibility condition (5), ensure that $\boldsymbol{u}_{\varepsilon} \in$ $\mathrm{W}^{2,2}(0, \mathrm{~T} ; H)$.

We shall show the existence and uniqueness of solution of problem $\mathrm{P}_{\varepsilon}$ by using the following slightly more general version of a direct constructive existence and uniqueness result obtained by J.M. Ricaud and E. Pratt [12].

Let $\left(H_{0},||.\right)$ and $\left(\mathrm{V}_{0},\|\|.\right)$ be two Hilbert spaces with the inner products denoted by $(.,$.$) and \langle.,$.$\rangle , respectively, such that \mathrm{V}_{0}$ is dense in $H_{0}$.

We set : $\mathcal{W}=\mathrm{W}^{1,2}\left(0, \mathrm{~T} ; \mathrm{V}_{0}\right) \cap \mathrm{W}^{2,2}\left(0, \mathrm{~T} ; H_{0}\right), \mathcal{K}=\left\{v \in \mathcal{W} ; v(0)=u_{0}\right.$, $\left.\dot{u}(0)=u_{1}\right\}$ with $u_{0}, u_{1} \in \mathrm{V}_{0}$.

Let $a, b: \mathrm{V}_{0} \times \mathrm{V}_{0} \rightarrow \mathbb{R}$ be two bilinear, symmetric, continuous and $\mathrm{V}_{0}$ elliptic forms in the following sense:

$\exists \mathrm{M}_{a}>0$ such that for all $v, w$ in $\mathrm{V}_{0} \times \mathrm{V}_{0}, a(v, w) \leq \mathrm{M}_{a}\|v\|\|w\|$,

$\exists \mathrm{M}_{b}>0$ such that for all $v, w$ in $\mathrm{V}_{0} \times \mathrm{V}_{0}, b(v, w) \leq \mathrm{M}_{b}\|v\|\|w\|$,

$\exists m_{a}>0$ such that for all $v$ in $\mathrm{V}_{0}, a(v, v) \geq m_{a}\|v\|^{2}$,

$\exists m_{b}>0$ such that for all $v$ in $\mathrm{V}_{0}, b(v, v) \geq m_{b}\|v\|^{2}$.

Let $\phi:[0, \mathrm{~T}] \times \mathrm{V}_{0}^{3} \rightarrow \mathbb{R}$ be a weakly continuous mapping such that for all $t \in[0, \mathrm{~T}],(u, v, w) \in \mathrm{V}_{0}^{3}, \phi(t, u, v, w)=\phi_{1}(u, v, w)+\phi_{2}(t, u, v, w)$, and

for all weakly convergent sequences $\left(u_{k}\right)$ such that $u_{k} \rightarrow u$ in $\mathcal{W}$,

$\liminf _{k \rightarrow \infty} \int_{0}^{T} \phi\left(t, u_{k}(t), \dot{u}_{k}(t), \dot{u}_{k}(t)\right) d t \geq \int_{0}^{\mathrm{T}} \phi(t, u(t), \dot{u}(t), \dot{u}(t)) d t$,

for all $u, v \in \mathrm{V}_{0}$ the mapping $\phi_{1}(u, v,$.$) is linear,$

$\phi_{2}\left(0, u_{0}, u_{1},.\right)=0, \phi_{2}(t, u, v,$.$) is a semi-norm,$

$\exists \eta>0$, such that $\forall t_{1}, t_{2} \in[0, \mathrm{~T}], \forall u_{1}, u_{2}, v_{1}, v_{2}, w_{1}, w_{2} \in \mathrm{V}_{0}$,

$\left|\phi\left(t_{1}, u_{1}, v_{1}, w_{1}\right)-\phi\left(t_{1}, u_{1}, v_{1}, w_{2}\right)+\phi\left(t_{2}, u_{2}, v_{2}, w_{2}\right)-\phi\left(t_{2}, u_{2}, v_{2}, w_{1}\right)\right|$

$\leq \eta\left(\left\|u_{1}-u_{2}\right\|+\left|v_{1}-v_{2}\right|+\left|t_{1}-t_{2}\right|\right)\left\|w_{1}-w_{2}\right\|$.

Let $f:[0, \mathrm{~T}] \rightarrow \mathrm{V}_{0}$ be in $\mathrm{W}^{1, \infty}\left(0, \mathrm{~T} ; \mathrm{V}_{0}\right)$. We assume that the initial conditions $u_{0}$ and $u_{1}$ verify the following compatibility condition: there exists $l \in H_{0}$ such that

$$
a\left(u_{0}, w\right)+b\left(u_{1}, w\right)+\phi_{1}\left(u_{0}, u_{1}, w\right)=\langle f(0), w\rangle+(l, w) \quad \forall w \in \mathrm{V}_{0} .
$$

We consider the following problem.

Problem $Q$ : Find $u \in \mathcal{K}$ such that for almost all $\mathrm{t} \in] 0, \mathrm{~T}[$ :

$$
\begin{aligned}
& (\ddot{u}(t), v-\dot{u}(t))+a(u(t), v-\dot{u}(t))+b(\dot{u}(t), v-\dot{u}(t))+\phi(t, u(t), \dot{u}(t), v) \\
& -\phi(t, u(t), \dot{u}(t), \dot{u}(t)) \geq\langle f(t), v-\dot{u}(t)\rangle \quad \forall v \in \mathrm{V}_{0} .
\end{aligned}
$$


Theorem 3.1. Assume that (8)-(16) hold. Then there exists a unique solution of problem $Q$.

The proof of this result is not presented here as it is similar to that in [12] , where the particular case of $\phi(t, u, v, w)$ independent of $v$ has been considered.

In order to solve problem $\mathrm{P}_{\varepsilon}$, we shall now use this theorem for $a, b$ of problem $\mathrm{P}_{\varepsilon}, \quad \mathrm{V}_{0}=\mathrm{V}, H_{0}=H, f=\mathbf{L}, \phi_{1}(\boldsymbol{u}, \boldsymbol{v}, \boldsymbol{w})=\int_{\Gamma_{c}} \frac{1}{\varepsilon}\left(u_{N}\right)_{+} w_{N} d s$ and $\phi_{2}(t, \boldsymbol{u}, \boldsymbol{v}, \boldsymbol{w})=\int_{\Gamma_{c}} \mu\left|(\mathcal{R} \boldsymbol{\sigma}(\boldsymbol{u}, \boldsymbol{v}))_{N}\right|\left|\boldsymbol{w}_{\mathrm{T}}\right| d s$, where $\boldsymbol{\sigma}(\boldsymbol{u}, \boldsymbol{v})=\mathbf{A} \boldsymbol{\epsilon}(\boldsymbol{u})+\mathbf{B} \boldsymbol{\epsilon}(\boldsymbol{v})$.

From the properties of $\operatorname{mes}\left(\Gamma_{u}\right)$, of the coefficients $\left(a_{i j k l}\right),\left(b_{i j k l}\right)$, and using Korn's inequality for the $\mathrm{V}$ - ellipticity, it follows that $a$ and $b$ satisfy (8)-(11). The properties (2) imply that $\mathbf{L} \in \mathcal{W}^{1, \infty}(0, \mathrm{~T} ; \mathrm{V})$ and (5) implies the compatibility condition (16). Using the properties of $\mathcal{R}$, one can easily obtain that (12)-(15) are satisfied. Thus by theorem 3.1 we obtain the following result.

Theorem 3.2. Under the above assumptions, for every $\varepsilon>0$ there exists a unique solution of problem $\mathrm{P}_{\varepsilon}$.

Now we may study the approximation of a solution of Signorini's problem with nonlocal friction law by a sequence of penalized solutions.

\section{Existence of a solution of unilateral contact problem}

We shall establish for $\boldsymbol{u}_{\varepsilon}$ several useful estimates, independent of $\varepsilon$, which will enable us to pass to the limit when $\varepsilon$ tends towards 0 . Firstly, let us prove that

$$
\begin{aligned}
& \dot{\boldsymbol{u}}_{\varepsilon} \text { is bounded in } \mathrm{L}^{\infty}(0, \mathrm{~T} ; H) \cap \mathrm{L}^{2}(0, \mathrm{~T} ; \mathrm{V}), \\
& \boldsymbol{u}_{\varepsilon} \text { is bounded in } \mathrm{L}^{\infty}(0, \mathrm{~T} ; \mathrm{V}), \\
& \frac{\left(u_{\epsilon N}\right)_{+}}{\sqrt{\epsilon}} \text { is bounded in } \mathrm{L}^{\infty}\left(0, \mathrm{~T} ; \mathrm{L}^{2}\left(\Gamma_{c}\right)\right),
\end{aligned}
$$

by positive constants depending on $\left\|\boldsymbol{u}_{\mathbf{0}}\right\|,\left\|\boldsymbol{u}_{\mathbf{1}}\right\|,\left|\mathbf{F}_{\mathbf{1}}\right|$ and $\left\|\mathbf{F}_{\mathbf{2}}\right\|$. By integrating (7) from 0 to $t$, for any $t$ in $] 0, \mathrm{~T}[$, we obtain

$$
\begin{gathered}
\int_{0}^{t}\left(\ddot{\boldsymbol{u}}_{\varepsilon}, \boldsymbol{v}-\dot{\boldsymbol{u}}_{\varepsilon}\right) d \tau+\int_{0}^{t} a\left(\boldsymbol{u}_{\varepsilon}, \boldsymbol{v}-\dot{\boldsymbol{u}}_{\varepsilon}\right) d \tau+\int_{0}^{t} b\left(\dot{\boldsymbol{u}}_{\varepsilon}, \boldsymbol{v}-\dot{\boldsymbol{u}}_{\varepsilon}\right) d \tau \\
+\int_{0}^{t} \int_{\Gamma_{c}} \frac{1}{\varepsilon}\left(u_{\varepsilon N}\right)_{+}\left(v_{N}-\dot{u}_{\varepsilon N}\right) d s d \tau+\int_{0}^{t} \int_{\Gamma_{c}} \mu\left|(\mathcal{R} \boldsymbol{\sigma})_{N}\right|\left(\left|\boldsymbol{v}_{\mathrm{T}}\right|-\left|\dot{\boldsymbol{u}}_{\varepsilon \mathrm{T}}\right|\right) d s d \tau \\
\geq \int_{0}^{t}\left\langle\mathbf{L}, \boldsymbol{v}-\dot{\boldsymbol{u}}_{\varepsilon}\right\rangle d \tau \quad \forall \boldsymbol{v} \in \mathrm{L}^{2}(0, \mathrm{~T} ; \mathrm{V})
\end{gathered}
$$


By taking $\boldsymbol{v}=\mathbf{0}$ we have

$$
\begin{aligned}
& \int_{0}^{t}\left(\ddot{\boldsymbol{u}}_{\varepsilon}, \dot{\boldsymbol{u}}_{\varepsilon}\right) d \tau+\int_{0}^{t} a\left(\boldsymbol{u}_{\varepsilon}, \dot{\boldsymbol{u}}_{\varepsilon}\right) d \tau+\int_{0}^{t} b\left(\dot{\boldsymbol{u}}_{\varepsilon}, \dot{\boldsymbol{u}}_{\varepsilon}\right) d \tau \\
+ & \int_{0}^{t} \int_{\Gamma_{c}} \frac{1}{\varepsilon}\left(u_{\varepsilon N}\right)_{+} \dot{u}_{\varepsilon N} d s d \tau+\int_{0}^{t} \int_{\Gamma_{c}} \mu\left|(\mathcal{R} \boldsymbol{\sigma})_{N}\right|\left|\dot{\boldsymbol{u}}_{\varepsilon \mathrm{T}}\right| d s d \tau \leq \int_{0}^{t}\left\langle\mathbf{L}, \dot{\boldsymbol{u}}_{\varepsilon}\right\rangle d \tau .
\end{aligned}
$$

We integrate the first two terms and find a minorant to the third one by using the $\mathrm{V}$ - ellipticity of $b$. Since the term $\int_{0}^{t} \int_{\Gamma_{c}} \frac{1}{\varepsilon}\left(u_{\varepsilon N}\right)_{+} \dot{u}_{\varepsilon N} d s d \tau$ can be written as $\int_{\Gamma_{c}} \frac{1}{2 \varepsilon}\left(u_{\varepsilon N}(t)\right)_{+}^{2} d s-\int_{\Gamma_{c}} \frac{1}{2 \varepsilon}\left(u_{\varepsilon N}(0)\right)_{+}^{2} d s$, we obtain the following result:

$$
\begin{aligned}
& \frac{1}{2}|\dot{\boldsymbol{u}}(t)|^{2}+\frac{1}{2} m_{a}\|\boldsymbol{u}(t)\|^{2}+c_{1} \int_{0}^{t}\|\dot{\boldsymbol{u}}\|^{2} d \tau+\frac{1}{2} \int_{\Gamma_{c}} \frac{1}{\varepsilon}\left(u_{\varepsilon N}(t)\right)_{+}^{2} d s \\
& \leq \frac{1}{2}\left|\boldsymbol{u}_{\mathbf{1}}\right|^{2}+\frac{1}{2} \mathrm{M}_{a}\left\|\boldsymbol{u}_{\mathbf{0}}\right\|^{2}+c_{2} \int_{0}^{t}\|\mathbf{L}\|^{2} d \tau,
\end{aligned}
$$

where we have used Schwarz's and Young's inequalities, the relation $\boldsymbol{u}_{\mathbf{0}} \in \mathrm{K}$ and where $c_{1}, c_{2}$ are positive constants which depend on the initial conditions, the given forces but not on $\varepsilon$. Thus (18)-(20) are established.

In order to pass to the limit when $\varepsilon$ tends towards 0 , we need an estimate on $\ddot{\boldsymbol{u}}_{\varepsilon}$ independent of $\varepsilon$. Choosing in $\mathrm{P}_{\varepsilon}, \boldsymbol{v}=\dot{\boldsymbol{u}}_{\varepsilon} \pm \boldsymbol{\psi}$, where $\boldsymbol{\psi} \in \mathrm{L}^{2}\left(0, \mathrm{~T} ; \boldsymbol{H}_{0}^{1}(\Omega)\right)$, we have

$$
\begin{aligned}
& \int_{0}^{\mathrm{T}}\left(\ddot{\boldsymbol{u}}_{\epsilon}, \boldsymbol{\psi}\right) d t+\int_{0}^{\mathrm{T}} a\left(\boldsymbol{u}_{\epsilon}, \boldsymbol{\psi}\right) d t+\int_{0}^{\mathrm{T}} b\left(\dot{\boldsymbol{u}}_{\epsilon}, \boldsymbol{\psi}\right) d t=\int_{0}^{\mathrm{T}}\left(\mathbf{F}_{\mathbf{1}}, \boldsymbol{\psi}\right) d t \\
& \forall \boldsymbol{\psi} \in \mathrm{L}^{2}\left(0, \mathrm{~T} ; \boldsymbol{H}_{0}^{1}(\Omega)\right) .
\end{aligned}
$$

Thus

$\ddot{\boldsymbol{u}}_{\epsilon}$ is bounded in $\mathrm{L}^{2}\left(0, \mathrm{~T} ; \boldsymbol{H}^{-1}(\Omega)\right)$ by a constant independent of $\varepsilon$.

Let $\boldsymbol{v}$ be in $\mathrm{L}^{\infty}(0, \mathrm{~T} ; \mathrm{V}) \cap \mathrm{W}^{1,2}(0, \mathrm{~T} ; H)$ with $\boldsymbol{v}(t) \in \mathrm{K}$ for all $t \in[0, \mathrm{~T}]$. If we integrate by parts the first term in $\mathrm{P}_{\varepsilon}$, then we have

$$
\begin{aligned}
& \left(\dot{\boldsymbol{u}}_{\varepsilon}(\mathrm{T}), \boldsymbol{v}(\mathrm{T})-\boldsymbol{u}_{\varepsilon}(\mathrm{T})\right)-\left(\boldsymbol{u}_{1}, \boldsymbol{v}(0)-\boldsymbol{u}_{\mathbf{0}}\right)-\int_{0}^{\mathrm{T}}\left(\dot{\boldsymbol{u}}_{\varepsilon}, \dot{\boldsymbol{v}}-\dot{\boldsymbol{u}}_{\varepsilon}\right) d t \\
& \quad+\int_{0}^{\mathrm{T}} a\left(\boldsymbol{u}_{\varepsilon}, \boldsymbol{v}-\boldsymbol{u}_{\varepsilon}\right) d t+\int_{0}^{\mathrm{T}} b\left(\dot{\boldsymbol{u}}_{\varepsilon}, \boldsymbol{v}-\boldsymbol{u}_{\varepsilon}\right) d t \\
& -\int_{0}^{\mathrm{T}} \int_{\Gamma_{c}} \mu\left|(\mathcal{R} \boldsymbol{\sigma})_{N}\right|\left(\left|\boldsymbol{v}_{\mathrm{T}}-\boldsymbol{u}_{\varepsilon \mathrm{T}}+\dot{\boldsymbol{u}}_{\varepsilon \mathrm{T}}\right|-\left|\dot{\boldsymbol{u}}_{\varepsilon \mathrm{T}}\right|\right) d s d t \geq \int_{0}^{\mathrm{T}}\left\langle\mathbf{L}, \boldsymbol{v}-\boldsymbol{u}_{\varepsilon}\right\rangle d t .
\end{aligned}
$$

We shall use the following compactness results due to J. Simon [13] (Corollary 4), which extend some classical results of J.P. Aubin and J.L. Lions. 
Theorem 4.1. Let $X, U$ and $Y$ be three Banach spaces such that $X \subset U \subset Y$ with compact imbedding $X \rightarrow U$.

Let $\mathcal{F}$ be bounded in $\mathrm{L}^{p}(0, \mathrm{~T} ; X)$, where $1 \leq p<\infty$, and $\partial \mathcal{F} / \partial t=\{\dot{f} ; f \in$ $\mathcal{F}\}$ be bounded in $\mathrm{L}^{1}(0, \mathrm{~T} ; Y)$. Then $\mathcal{F}$ is relatively compact in $\mathrm{L}^{p}(0, \mathrm{~T} ; U)$.

Let $\mathcal{F}$ be bounded in $\mathrm{L}^{\infty}(0, \mathrm{~T} ; X)$ and $\partial \mathcal{F} / \partial t$ be bounded in $\mathrm{L}^{r}(0, \mathrm{~T} ; Y)$, where $r>1$. Then $\mathcal{F}$ is relatively compact in $\mathrm{C}([0, \mathrm{~T}], U)$.

First, we consider the following sets:

$$
X=\mathrm{V}, Y=H, r=\infty, \mathcal{F}=\left(\boldsymbol{u}_{\varepsilon}\right) .
$$

Since the imbedding $\boldsymbol{H}^{s_{1}}(\Omega) \rightarrow \boldsymbol{H}^{s_{2}}(\Omega)$, with $s_{1}>s_{2}$, is compact (see, e.g. J.L. Lions and E. Magenes [9]) and we have established $(18,19)$, by theorem 4.1 it follows that

$$
\left(\boldsymbol{u}_{\varepsilon}\right) \text { is relatively compact in } \mathrm{C}\left([0, \mathrm{~T}], \boldsymbol{H}^{1 / 2}(\Omega)\right) .
$$

Now if we consider :

$$
X=H, U=\boldsymbol{H}^{-1 / 2}(\Omega), Y=\boldsymbol{H}^{-1}(\Omega), r=\infty \mathcal{F}=\left(\dot{\boldsymbol{u}}_{\varepsilon}\right),
$$

then, with the estimates $(18,24)$, by Simon's theorem 4.1 we obtain that

$$
\left(\dot{\boldsymbol{u}}_{\varepsilon}\right) \text { is relatively compact in } \mathrm{C}\left([0, \mathrm{~T}], \boldsymbol{H}^{-1 / 2}(\Omega)\right) \text {. }
$$

Using again theorem 4.1 for :

$$
X=\mathrm{V}, U=H, Y=\boldsymbol{H}^{-1}(\Omega), p=2, \mathcal{F}=\left(\dot{\boldsymbol{u}}_{\varepsilon}\right),
$$

it follows that

$$
\left(\dot{\boldsymbol{u}}_{\varepsilon}\right) \text { is relatively compact in } \mathrm{L}^{2}(0, \mathrm{~T} ; H) .
$$

Thus we have the following result.

Lemma 4.1. There exists a subsequence of $\left(\boldsymbol{u}_{\varepsilon}\right)$, still denoted by $\left(\boldsymbol{u}_{\varepsilon}\right)$, and an element $\boldsymbol{u} \in \mathrm{C}^{1}\left([0, \mathrm{~T}], \boldsymbol{H}^{-1 / 2}(\Omega)\right)$, such that

$$
\begin{aligned}
& \boldsymbol{u}_{\varepsilon} \rightarrow \boldsymbol{u} \text { in } \mathrm{C}\left([0, \mathrm{~T}], \boldsymbol{H}^{1 / 2}(\Omega)\right), \\
& \dot{\boldsymbol{u}}_{\varepsilon} \rightarrow \dot{\boldsymbol{u}} \text { in } \mathrm{C}\left([0, \mathrm{~T}], \boldsymbol{H}^{-1 / 2}(\Omega)\right), \\
& \dot{\boldsymbol{u}}_{\varepsilon} \rightarrow \dot{\boldsymbol{u}} \text { in } \mathrm{L}^{2}(0, \mathrm{~T} ; H)
\end{aligned}
$$

From $(18,19)$ it follows that the subsequence $\left(\boldsymbol{u}_{\varepsilon}\right)$ can be chosen such that

$$
\begin{aligned}
& \boldsymbol{u}_{\varepsilon} \rightarrow \boldsymbol{u} \text { weak } * \text { in } \mathrm{L}^{\infty}(0, \mathrm{~T} ; \mathrm{V}), \\
& \dot{\boldsymbol{u}}_{\varepsilon} \rightarrow \boldsymbol{u} \text { in } \mathrm{L}^{2}(0, \mathrm{~T} ; \mathrm{V})
\end{aligned}
$$

which imply

$$
\boldsymbol{u}_{\varepsilon} \rightarrow \boldsymbol{u} \text { in } \mathrm{W}^{1,2}(0, \mathrm{~T} ; \mathrm{V})
$$


Using the relation $\mathrm{W}^{1,2}(0, \mathrm{~T} ; \mathrm{V}) \subset \mathrm{C}([0, \mathrm{~T}], \mathrm{V})$, we have that $\left(\boldsymbol{u}_{\varepsilon}(t)\right)$ is bounded in $\mathrm{V}$ for all $t$ in $[0, \mathrm{~T}]$. Let $\mathrm{E}$ be a countable dense subset of $[0, \mathrm{~T}]$. By a diagonal process, we can extract a subsequence, still denoted by $\left(\boldsymbol{u}_{\varepsilon}\right)$, such that for all $s \in \mathrm{E},\left(\boldsymbol{u}_{\varepsilon}(s)\right)$ converges weakly to $\boldsymbol{u}(s)$ and by similar arguments as in [1], we obtain

$$
\forall t \in[0, \mathrm{~T}], \quad \boldsymbol{u}_{\varepsilon}(t) \rightarrow \boldsymbol{u}(t) \text { in } \mathrm{V} .
$$

The next convergence result will enable us to pass to the limit in the friction term (see also [7]).

Lemma 4.2. We have

$$
\dot{\boldsymbol{u}}_{\varepsilon} \rightarrow \dot{\boldsymbol{u}} \text { in } \mathrm{L}^{2}\left(0, \mathrm{~T} ; \mathbf{L}^{2}\left(\Gamma_{c}\right)\right)
$$

Proof. Let $U$ be a Banach space such that the imbedding $\mathrm{V} \rightarrow U$ is compact and there exists a bounded linear operator $\mathcal{T}: U \rightarrow \mathrm{L}^{2}\left(\Gamma_{c}\right)$.

Then, using (34) and theorem 4.1 for $X=\mathrm{V}, U, Y=\boldsymbol{H}^{-1}(\Omega), p=2, \mathcal{F}=$ $\left(\dot{\boldsymbol{u}}_{\varepsilon}\right)$, we obtain that $\dot{\boldsymbol{u}}_{\varepsilon} \rightarrow \dot{\boldsymbol{u}}$ in $\mathrm{L}^{2}(0, \mathrm{~T} ; U)$ and the conclusion follows.

We may now show the following existence result for problem $\mathrm{P}$.

Theorem 4.2. Let $\boldsymbol{u}_{\mathbf{0}}, \boldsymbol{u}_{\mathbf{1}}$, and $\mathbf{F}_{\mathbf{2}}$ be given satisfying (2). Then there exists a subsequence of solutions $\left(\boldsymbol{u}_{\varepsilon}\right)$ of the penalized problems which converges to a solution $\boldsymbol{u}$ of dynamic Signorini's problem with nonlocal friction $\mathrm{P}$.

Proof. It is clear that $\boldsymbol{u}(0)=\boldsymbol{u}_{\mathbf{0}}, \dot{\boldsymbol{u}}(0)=\boldsymbol{u}_{\mathbf{1}}$. From (35) and the compactness of the imbedding $\boldsymbol{H}^{1 / 2}(\Gamma) \rightarrow \mathbf{L}^{2}(\Gamma)$, we have

$$
\forall t \in] 0, \mathrm{~T}\left[, u_{\varepsilon N} \rightarrow u_{N} \text { in } \mathrm{L}^{2}\left(\Gamma_{c}\right),\right.
$$

which with (20) imply

$$
0=\liminf \left\|\left(u_{\epsilon N}\right)_{+}\right\|_{\mathrm{L}^{2}\left(\Gamma_{c}\right)}^{2} \geq\left\|\left(u_{N}\right)_{+}\right\|_{\mathrm{L}^{2}\left(\Gamma_{c}\right)}^{2} .
$$

Hence for all $t \in] 0, \mathrm{~T}[, \boldsymbol{u}(t) \in \mathrm{K}$.

We now write inequality (25) as follows:

$$
\begin{aligned}
& \left(\dot{\boldsymbol{u}}_{\varepsilon}(\mathrm{T}), \boldsymbol{v}(\mathrm{T})-\boldsymbol{u}_{\varepsilon}(\mathrm{T})\right)-\left(\boldsymbol{u}_{\mathbf{1}}, \boldsymbol{v}(0)-\boldsymbol{u}_{\mathbf{0}}\right)-\int_{0}^{\mathrm{T}}\left(\dot{\boldsymbol{u}}_{\varepsilon}, \dot{\boldsymbol{v}}-\dot{\boldsymbol{u}}_{\varepsilon}\right) d t+\int_{0}^{\mathrm{T}} a\left(\boldsymbol{u}_{\varepsilon}, \boldsymbol{v}\right) d t \\
& +\int_{0}^{\mathrm{T}} b\left(\dot{\boldsymbol{u}}_{\varepsilon}, \boldsymbol{v}\right) d t-\int_{0}^{\mathrm{T}} \int_{\Gamma_{c}} \mu\left|(\mathcal{R} \boldsymbol{\sigma})_{N}\right|\left(\left|\boldsymbol{v}_{\mathrm{T}}-\boldsymbol{u}_{\varepsilon \mathrm{T}}+\dot{\boldsymbol{u}}_{\varepsilon \mathrm{T}}\right|-\left|\dot{\boldsymbol{u}}_{\varepsilon \mathrm{T}}\right|\right) d s d t \\
& \geq \int_{0}^{\mathrm{T}} a\left(\boldsymbol{u}_{\varepsilon}, \boldsymbol{u}_{\varepsilon}\right) d t+\frac{1}{2} b\left(\boldsymbol{u}_{\varepsilon}(\mathrm{T}), \boldsymbol{u}_{\varepsilon}(\mathrm{T})\right)-\frac{1}{2} b\left(\boldsymbol{u}_{\mathbf{1}}, \boldsymbol{u}_{\mathbf{1}}\right)+\int_{0}^{\mathrm{T}}\left\langle\mathbf{L}, \boldsymbol{v}-\boldsymbol{u}_{\varepsilon}\right\rangle d t \\
& \quad \forall \boldsymbol{v} \in \mathrm{L}^{\infty}(0, \mathrm{~T} ; \mathrm{V}) \cap \mathrm{W}^{1,2}(0, \mathrm{~T} ; H) \text { with } \boldsymbol{v}(t) \in \mathrm{K} \text { for all } t \in[0, \mathrm{~T}] .
\end{aligned}
$$

Note that, using lemma 4.1, (32) and (33), we may pass to the limit when $\varepsilon$ tends towards 0 in the first four terms, containing $\varepsilon$, in the left-hand side of (37). We 
may also pass to the limit in the friction term using the property (3) of $\mathcal{R}$ and lemma 4.2. In the right-hand side, the elastic and the viscous terms are convex and continuous on $\mathrm{L}^{2}(0, \mathrm{~T} ; \mathrm{V})$ and respectively $\mathrm{V}$, and hence weakly sequentially lower semi-continuous.

Finally, from the convergence properties of the terms in the left-hand side, using (32), (35) and computing lim of the left-hand side of (37) and lim inf of the right-hand side, we obtain

$$
\begin{aligned}
& (\dot{\boldsymbol{u}}(\mathrm{T}), \boldsymbol{v}(\mathrm{T})-\boldsymbol{u}(\mathrm{T}))-\left(\boldsymbol{u}_{\mathbf{1}}, \boldsymbol{v}(0)-\boldsymbol{u}_{\mathbf{0}}\right)-\int_{0}^{\mathrm{T}}(\dot{\boldsymbol{u}}, \dot{\boldsymbol{v}}-\dot{\boldsymbol{u}}) d t+\int_{0}^{\mathrm{T}} a(\boldsymbol{u}, \boldsymbol{v}) d t \\
& +\int_{0}^{\mathrm{T}} b(\dot{\boldsymbol{u}}, \boldsymbol{v}) d t-\int_{0}^{\mathrm{T}} \int_{\Gamma_{c}} \mu\left|(\mathcal{R} \boldsymbol{\sigma})_{N}\right|\left(\left|\boldsymbol{v}_{\mathrm{T}}-\boldsymbol{u}_{\mathrm{T}}+\dot{\boldsymbol{u}}_{\mathrm{T}}\right|-\left|\dot{\boldsymbol{u}}_{\mathrm{T}}\right|\right) d s d t \\
& \geq \int_{0}^{\mathrm{T}} a(\boldsymbol{u}, \boldsymbol{u}) d t+\frac{1}{2} b(\boldsymbol{u}(\mathrm{T}), \boldsymbol{u}(\mathrm{T}))-\frac{1}{2} b\left(\boldsymbol{u}_{1}, \boldsymbol{u}_{\mathbf{1}}\right)+\int_{0}^{\mathrm{T}}\langle\mathbf{L}, \boldsymbol{v}-\boldsymbol{u}\rangle d t \\
& \quad \forall \boldsymbol{v} \in \mathrm{L}^{\infty}(0, \mathrm{~T} ; \mathrm{V}) \cap \mathrm{W}^{1,2}(0, \mathrm{~T} ; H) \text { with } \boldsymbol{v}(t) \in \mathrm{K} \text { for all } t \in[0, \mathrm{~T}]
\end{aligned}
$$

Thus $\boldsymbol{u}$ is a solution of problem $\mathrm{P}$.

\section{References}

[1] M. Cocou and J.M. Ricaud, Analysis of a class of implicit evolution inequalities associated to viscoelastic dynamic contact problems with friction, Int. J. Engrg. Sci. 38 (2000), 1535-1552.

[2] G. Duvaut and J.L. Lions, Les inéquations en mécanique et en physique. Dunod, Paris 1972.

[3] I. Figueiredo and L. Trabucho, A class of contact and friction dynamic problems in thermoelasticity and in viscoelasticity, Int. J. Engrg. Sci. 33 (1995), 45-66.

[4] J. Jarušek, Dynamical contact problems for bodies with a singular memory, Boll. Unione Mat. Ital. 7 (9-A) (1995), 581-592.

[5] J.U. Kim, A boundary thin obstacle problem for a wave equation, Commun. in Partial Differential Equations 14 (1989), 1011-1026.

[6] K. L. Kuttler, Dynamic friction contact problems for general normal and friction laws, Nonlinear Analysis, Theory, Meth. Applic. 28 (1997), 559-575.

[7] K. L. Kuttler and M. Shillor, Dynamic bilateral contact with discontinuous friction coefficient, Nonlinear Analysis, Theory, Meth. Applic. 45 (2001), 309-327.

[8] G. Lebeau and M. Schatzman, A wave problem in a half-space with a unilateral constraint at the boundary, J.Diff.Eqs. 53 (1984), 309-361.

[9] J.L. Lions and E. Magenes, Problèmes aux limites non homogènes. Dunod, Paris 1967.

[10] J.A.C. Martins and J.T. Oden, Existence and uniqueness results for dynamic contact problems with nonlinear normal friction and interface laws, Nonlinear Analysis, Theory, Meth. Applic. 11 (1987), 407-428.

[11] J. Muñoz-Rivera and R. Racke, Multidimensional contact problems in thermoelasticity, Siam J. Appl. Math. 58 (1998), 1307-1337.

[12] J.M. Ricaud and E. Pratt, Analysis of a time discretisation for an implicit variational inequality modelling dynamic contact problems with friction, Math. Meth. Appl. Sci. 24 (2000), 491-511.

[13] J. Simon, Compact sets in the space L ${ }^{p}(0, T ; B)$, Ann. Mat. Pura Appl. 146 (1987), 65-96. 